\title{
Increased Urinary N-acetyl-beta-D-glucosaminidase Activity in Children with Hydronephrosis
}

\author{
Sylva Skalova, Pavel Rejtar, Stepan Kutilek
}

Departments of Pediatrics and Radiology, Charles University in Prague, Faculty of Medicine in Hradec Kralove, Czech Republic, and Center for Clinical and Basic Research, Pardubice, Czech Republic

\begin{abstract}
Objective: Hydronephrosis leads to deterioration of renal function. As urinary N-acetyl-beta-D-glucosaminidase (U-NAG) activity is considered a sensitive marker of renal tubular impairment, our aim was to measure U-NAG in children with hydronephrosis and to look for a relationship among selected clinical parameters.

Materials and Methods: We studied 31 children ( 22 boys and 9 girls, mean age $2.3 \pm 2.5$ years) with hydronephrosis grade 1-4 that had U-NAG/creatinine ratio (U-NAG/Cr) measured.

Results: The U-NAG/Cr was significantly higher in patients with hydronephrosis compared to reference data $(\mathrm{p}=0.002)$. There was no difference in U-NAG/Cr between children with unilateral and bilateral hydronephrosis $(\mathrm{p}=0.51)$. There was no significant difference in U-NAG/Cr between children with grades 1-3 (pooled data) and grade 4, respectively $(\mathrm{p}=0.89)$. There was no correlation between U-NAG/Cr and the grade of hydronephrosis $(r=0.01)$.

Conclusions: U-NAG/Cr is increased in children with hydronephrosis grade 1-4, and there is no relationship with the grade of hydronephrosis. U-NAG is a useful marker of renal tubular dysfunction, however its relationship with the degree of kidney damage in patients with hydronephrosis should be considered as doubtful.
\end{abstract}

Key words: children; hydronephrosis; $N$ acetyl beta d glucosaminidase

Int Braz. J Urol. 2007; 33: 80-6

\section{INTRODUCTION}

Hydronephrosis leads to deterioration of renal function $(1,2)$. N-acetyl-beta-D-glucosaminidase (NAG) is a lysosomal enzyme, which is abundantly present in the cells of the proximal tubule and is considered as a very sensitive marker of renal tubular impairment in various disease states $(3,4)$. Our aim was to measure urinary NAG activity (U-NAG) in children with hydronephrosis and to look for a possible relationship between patients' clinical data and U-NAG.

\section{MATERIALS AND METHODS}

We studied 31 children ( 22 boys and 9 girls, mean age $2.25 \pm 2.50$ years; range $0.08-9.08$ y) with hydronephrosis. Informed consent was obtained from parents of each patient prior to any procedures described in this paper. Hydronephrosis was diagnosed by means of abdominal ultrasonography either prenatally $(n=20)$ or postnatally $(n=11)$, the latter at the mean age of $6 \pm 14$ months (range 0.1 - 48 months). In all patients, the hydronephrosis and its grade was further evaluated postnatally by means of ultrasound 
and ${ }^{99 \mathrm{~m}} \mathrm{Tc}$ mercaptoacetyltriglycine (MAG3) "well tempered" renography $(5,6)$. Hydronephrosis was graded according to the Society for Fetal Urology (SFU) classification (1). Vesicoureteral reflux was ruled out in all patients by voiding cystourethrography. None of the patients had solitary kidney.

In 18 patients the hydronephrosis was unilateral, grade 1-4 (mean $3.1 \pm 0.8$ ), and in 13 patients, the hydronephrosis was bilateral, grade 1-4 (mean 2.9 \pm 0.7 ). In the patients with bilateral hydronephrosis and different grade on each side, the highest grade was taken into consideration. Therefore, the diagnostic distribution was as follows: grade $1, \mathrm{n}=1$; grade $2, \mathrm{n}=2$; grade $3, \mathrm{n}=16$; grade $4, \mathrm{n}=12$. All patients had their kidney functions evaluated by the "welltempered" diuretic renogram with ${ }^{99 m}$ Tc MAG3 (57). The relative renal function, expressed as percentage represented by the contribution of each kidney to the global renal function was evaluated. In only 2 children with unilateral hydronephrosis, the relative function of the affected kidney was $35 \%$. In the remaining 17 children with unilateral hydronephrosis, the relative function of the affected kidney exceeded $40 \%$. The mean value of the relative function of the affected kidney in the 19 patients with unilateral hydronephrosis was $47.3 \%$. In the entire group of 31 children, the mean relative renal function of the right and left kidney was 50.4\%: 49.6\%. In patients with hydronephrosis grade 1-3 there were no signs of obstruction, while obstruction was present in patients with grade 4 . The obstruction was evidenced by several criteria, such as progressive dilatation of the calyces and pelvis on ultrasound imaging; $>5 \%$ decrease per year in the function of hydronephrotic kidney on ${ }^{99 m}$ Tc MAG3 renogram; obstructive pattern of renogram curve after administration of furosemide with a clearance half-life greater than 20 minutes (5-7).

None of the patients underwent any surgical procedure due to hydronephrosis prior to the U-NAG measurements. Patients with grade 4 were later confined to surgical treatment.

All patients had their U-NAG and serum and urinary concentrations of creatinine (S-Cr, U-Cr) evaluated. None of the patients suffered from pyelonephritis at the time of the U-NAG/Cr and S-Cr evaluation. All patients were free from infection at least 4 months prior to the U-NAG/Cr and S-Cr evaluation. Urinary NAG was evaluated in the spot urine, collected after the first morning void. The blood and spot urine were collected either at the time of the ultrasonographic examination or in a period of \pm 1 month within abdominal ultrasonography and ${ }^{99 \mathrm{~m}} \mathrm{Tc}$ MAG3 renography. The influence of endogenous enzyme inhibitors was eliminated by diluting the urine specimens' 20 -fold. The urinary catalytic activity of NAG was then determined by fluorimetric assay. The S-Cr and U-Cr were estimated by Jaffe's kinetic method on Modular Analyser (Roche Diagnostics GmbH, Mannheim, Germany). The S-Cr values were expressed in $\mu \mathrm{mol} / \mathrm{L}$. The U-NAG values were expressed as the urinary NAG/creatinine (U-NAG/Cr) ratio in nkat/L : $\mathrm{mmol} / \mathrm{L}$. To eliminate the influence of age, the obtained results of S-Cr and U-NAG/Cr were expressed as standard deviation scores (SDS) or Z-scores by the equation SDS = (actual individual value - mean value for age) /standard deviation for age with the use of agerelated laboratory reference data for $\mathrm{S}-\mathrm{Cr}$ and previously obtained reference data for U-NAG/Cr (4). These reference standards of U-NAG/Cr were obtained from a total of 262 children (aged 0-18 years), and in particular from 213 children aged 0-10 years (4). The obtained values were compared to the age-related reference data and correlated with grade of hydronephrosis. The presence of either unilateral or bilateral hydronephrosis was also taken into consideration.

The statistical analysis was performed by ttest. The linear regression analysis was performed to compare the relationship among respective parameters. For all results, a p-value $<0.05$ was required for statistical significance.

\section{RESULTS}

The U-NAG/Cr values were significantly higher in the patients with hydronephrosis in comparison to the reference data (Table-1). There was no difference in U-NAG/Cr between children with unilateral and bilateral hydronephrosis $(\mathrm{p}=0.51)$.

As there were low patient numbers with hydronephrosis grade 1-2, we pooled the U-NAG/Cr data for this group of children together with hydronephro- 
Table 1-U-NAG/Cr and S-Cr values expressed as Z-scores $\pm S D$.

\begin{tabular}{lccl}
\hline Parameter & Mean & SD & p Value $\dagger$ \\
\hline U-NAG/Cr (grade 1-4) & 4.92 & 5.38 & 0.002 \\
U-NAG/Cr (grade 1-3) & 5.02 & 5.29 & 0.0006 \\
$\mathrm{U}-\mathrm{NAG/Cr}$ (grade 4) & 4.76 & 5.74 & 0.015 \\
$\mathrm{~S}-\mathrm{Cr}$ (grade 1-4) & 0.53 & 1.09 & 0.05 \\
\hline
\end{tabular}

U-NAG/Cr (grade 1-4), data from patients with hydronephrosis grade 1-4 $(n=31)$; $U-N A G / C r$ (grade $1-3)$, pooled data from patients with grade 1-3 $(n=19) ; U-N A G / C r$ (grade 4$)$, data from patients with grade $4(n=12) ; \mathrm{S}$ - $\mathrm{Cr}$ (grade 1-4), data from patients with hydronephrosis grade 1-4; † compared to reference data.

sis grade 3 . When compared to reference data, patients with grade $1-3(n=19)$ and those with grade $4(n=12)$ had significantly higher U-NAG/Cr activity (Table-1). However there was no significant difference in U-NAG/ Cr between children with grade 1-3 and grade 4, respectively $(\mathrm{p}=0.89)$. Neither was there any significant difference in the U-NAG/Cr values between children with unilateral and bilateral hydronephrosis when stratified for grade (grade 1-3 and 4, respectively; $p=0.55$ and $p$ $=0.50$, respectively). The $\mathrm{S}-\mathrm{Cr}$ was within $\pm 2 \mathrm{SD}$ range in 30/31 patients, however this was still significantly higher in comparison to reference data (Table-1). There was no difference in $\mathrm{S}-\mathrm{Cr}$ between children with unilateral and bilateral hydronephrosis $(\mathrm{p}=0.82)$. No correlations were observed between $\mathrm{U}-\mathrm{NAG} / \mathrm{Cr}$ and the grade of hydronephrosis $(\mathrm{r}=0.01)$, or between $\mathrm{S}-\mathrm{Cr}$ and the grade of hydronephrosis $(\mathrm{r}=-0.07)$. We found a positive correlation between $\mathrm{U}-\mathrm{NAG} / \mathrm{Cr}$ and $\mathrm{S}-\mathrm{Cr}$, which reached statistical significance $(r=0.40, p=0.05)$.

\section{COMMENTS}

The high values of U-NAG/Cr in our patients with hydronephrosis suggest renal tubular impairment and are in accordance with previously reported results, which are only scarce (8-13). Experimental studies revealed high U-NAG in rats with partial ureteral obstruction and hydronephrotic atrophy $(8,9)$. Increased U-NAG was detected in urine obtained from renal pelvis $(10,11)$ and bladder $(11)$, with pelvic U-
NAG levels higher than bladder U-NAG levels (11) in children with unilateral hydronephrosis. High U$\mathrm{NAG/Cr}$ levels were observed in children with renal pyelectasis (12). Interestingly, post-operative increase in U-NAG levels was reported in patients with hydronephrosis (13).

In our patients, the U-NAG/Cr values, measured in the spontaneously voided urine, were increased, regardless whether there was unilateral or bilateral hydronephrosis. Previously published observations based on evaluation of isotope renal function and imaging procedures gave evidence that children with grade 4 , and some with grade 3 of hydronephrosis, have impaired renal functions and should be confined to surgical treatment, which has been proven as beneficial $(1,2,14,15)$. It was therefore of particular interest to see if U-NAG was somehow related to the grade of hydronephrosis. However, the high levels of U-NAG did not correspond to the ultrasonographic degree of renal damage, as there was no correlation between U-NAG and the grade of hydronephrosis, and there was no difference in U-NAG between grades 1-3 and 4, respectively. Similarly, the renal functions, as assessed by the ${ }^{99 \mathrm{~m}} \mathrm{Tc}$ MAG3 renography, were not severely impaired. There was no difference in UNAG/Cr between children with unilateral and bilateral hydronephrosis. These results might suggest that the renal function, as assessed by ${ }^{99 \mathrm{~m}} \mathrm{Tc}$ MAG3 renography might not be solely related to the grade of hydronephrosis, and that U-NAG in hydronephrosis does not depend on the amount of affected renal tissue. Furthermore, we cannot rule out that the U-NAG can reflect even very mild changes in renal tubular function, which might occur even in low-grade non-obstructive hydronephrosis. There was a mild elevation of S-Cr, which reached statistical significance, and there was also a mild correlation between U-NAG/ $\mathrm{Cr}$ and $\mathrm{S}-\mathrm{Cr}$. However, the changes in $\mathrm{S}-\mathrm{Cr}$ in our group of patients are strongly obscured by the fact that all but one $\mathrm{S}-\mathrm{Cr}$ values remained within the \pm 2 SD range and that there was no difference between unilateral and bilateral hydronephrosis.

In conclusion, U-NAG/Cr is increased in children with hydronephrosis grade 1-4, however, there is no relationship with the grade of hydronephrosis or with the amount of affected renal tissue. U-NAG/ 
$\mathrm{Cr}$ is a useful marker of renal tubular impairment, however its relationship with the degree of kidney damage in patients with hydronephrosis should be considered as doubtful.

\section{ACKNOWLEDGEMENTS}

Prof. V. Palicka and his team from the Institute of Clinical Biochemistry and Diagnosis at the Faculty of Medicine in Hradec Králové performed the $\mathrm{S}-\mathrm{Cr}$ and $\mathrm{U}-\mathrm{NAG} / \mathrm{Cr}$ analyses.

\section{CONFLICT OF INTEREST}

None declared.

\section{REFERENCES}

1. Maizels M, Mitchell B, Kass E, Fernbach SK, Conway $\mathrm{JJ}$ : Outcome of nonspecific hydronephrosis in the infant: a report from the Registry of the Society for Fetal Urology. J Urol. 1994; 152: 2324-7.

2. Rodriguez LV, Lock J, Kennedy WA, Shortliffe LM: Evaluation of sonographic renal parenchymal area in the management of hydronephrosis. J Urol. 2001; 165: 548-51.

3. Csathy L, Pocsi I: Urinary N-acetyl-beta-Dglucosaminidase determination in newborns and children: methods and diagnostic applications. Eur J Clin Chem Clin Biochem. 1995; 33: 575-87.

4. Skalova S, Chladek J: Urinary N-acetyl-beta-Dglucosaminidase activity in healthy children. Nephrology (Carlton). 2004; 9: 19-21.

5. Conway JJ, Maizels M: The "well tempered" diuretic renogram: a standard method to examine the asymptomatic neonate with hydronephrosis or hydroureteronephrosis. A report from combined meetings of The Society for Fetal Urology and members of The Pediatric Nuclear Medicine Council-The Society of Nuclear Medicine. J Nucl Med. 1992; 33: 2047-51.

6. Rossleigh MA: Renal cortical scintigraphy and diuresis renography in infants and children. J Nucl Med. 2001; 42: 91-5.

7. Saremi F, Jadvar H, Siegel ME: Pharmacologic interventions in nuclear radiology: indications, imaging protocols, and clinical results. Radiographics. 2002; 22: 477-90.
8. Huland H, Gonnermann D, Werner B, Possin U: A new test to predict reversibility of hydronephrotic atrophy after stable partial unilateral ureteral obstruction. J Urol. 1988; 140: 1591-4.

9. Everaert K, Van de Wiele C, Delanghe J, Vander Eecken H, Van Haelst JP, Van de Voorde J, et al.: Urinary excretion of tubular proteins and the technetium99m dimercaptosuccinic acid (DMSA) absolute renal uptake in partial ureteral obstruction in rats: a functional evaluation of hydronephrotic kidneys. Urol Res. 1999; 27: 127-33.

10. Konda R, Orikasa S, Sakai K, Kuji S, Ota S, Kaneda $\mathrm{T}$, et al.: Evaluation of renal function and prediction of renal functional recovery in children with unilateral hydronephrosis using renal pelvic urine. Nippon Hinyokika Gakkai Zasshi. 1992; 83: 1815-22.

11. Carr MC, Peters CA, Retik AB, Mandell J: Urinary levels of the renal tubular enzyme N-acetyl-beta-Dglucosaminidase in unilateral obstructive uropathy. J Urol. 1994; 151: 442-5.

12. Leon Gonzalez J, Garcia Nieto V, Hernandez Rodriguez A, Fernandez Gonzalez L: Study of renal function in infants diagnosed with renal pyelectasis in the first year of life. An Esp Pediatr. 2001; 54: 458-62.

13. Ueda K, Kato J, Seki T: Urinary excretion of N-acetylbeta-D-glucosaminidase in patients with urological disease: with special reference to hydronephrosis. Hinyokika Kiyo. 1984; 30: 877-82.

14. Palmer LS, Maizels M, Cartwright PC, Fernbach SK, Conway JJ: Surgery versus observation for managing obstructive grade 3 to 4 unilateral hydronephrosis: a report from the Society for Fetal Urology. J Urol. 1998; 159: 222-8.

15. Sibai H, Salle JL, Houle AM, Lambert R: Hydronephrosis with diffuse or segmental cortical thinning: impact on renal function. J Urol. 2001; 165: 2293-5.

Accepted after revision:

November 1, 2006

\section{Correspondence address:}

Dr. Sylva Skálová

Department of Pediatrics

Charles University in Prague

Faculty of Medicine in Hradec Králové

Czech Republic

Fax: + 420 49583-2030

E-mail: skalova.s@seznam.cz 


\section{EDITORIAL COMMENT}

Congenital obstructive nephropathy represents a major cause of renal insufficiency in infants and children. At present, two puzzling issues of congenital hydronephrosis still need to be elucidated. One is the diagnosis of obstruction (distinguishing an obstructed from a nonobstructed collecting system), and the other is the existence and definition of a no-return point of renal damage. It is our aim to find a urinary biomarker aids in the diagnosis of renal tubular damage and medical therapy is given to protect renal function and accelerate its recovery after intervention.

NAG excretion in urine is widely used as a marker of tubular and glomerular injury in differential pathological states in human diseases. The authors measured urinary NAG in children with hydronephrosis and assert increased U-NAG/Cr in children with hydronephrosis grade 1-4 (although there were no signs of obstruction in patients with hydronephrosis grade 1-3), but there is no relationship with the grade of hydronephrosis. The increase of UNAG in children with unobstructed renal pyelectasis raise a question that if there is renal damage in children only with renal pyelectasis. A recent paper which showed significant discordance between conventional

\section{EDITORIAL COMMENT}

The authors investigated the urinary secretion of N-acetyl-beta-D-glucosaminidase (U-NAG) in the patients with unilateral and bilateral hydronephrosis in order to look at the relation between the severity of the U-NAG secretion and the grade of hydronephrosis. They convincingly show that there is an increased secretion of U-NAG in children with hydronephrosis due to ureteropelvic junction (UPJ) obstruction reflecting proximal tubular injury in these patients. However, they failed to demonstrate the significant relation between the degree of hydronephrosis and renal damage and U-NAG secretion, therefore eliminating the utilization of this imaging and histological findings in congenital ureteropelvic junction obstruction perhaps could answer this question (1). However, it also needs longterm follow up to see if the children with unobstructed obstruction have the risk for progressive renal damage. It is exciting if a prognostic factor indicating renal damage in children with congenital hydronephrosis can be confirmed by subsequent studies. More thoughtful work is needed to make this a reality.

\section{REFERENCE}

1. Huang WY, Peters CA, Zurakowski D, Borer JG, Diamond DA, Bauer SB, et al.: Renal biopsy in congenital ureteropelvic junction obstruction: evidence for parenchymal maldevelopment. Kidney Int. 2006; 69:137-43.

Dr. Y. Yang

Department of Pediatric Surgery China Medical University

Shenyang City, China

E-mail: yangxy70@hotmail.com

marker in the decision making process for surgery in patients with antenatal hydronephrosis. Most urologists manage the majority of the cases of fetal hydronephrosis due to UPJ obstruction by nonoperative observation, reserving surgery only for patients with deterioration of renal function or clinical symptoms. However the natural history of fetal hydronephrosis, the optimal time for surgery, the ability to define which kidney will benefit from surgical intervention, and which children will have deterioration in renal function while on surveillance, is still a matter of controversy. We have recently published our experience regarding predictive factors 
for surgery in children with antenatal diagnosis of hydronephrosis, which led to postnatal diagnosis of UPJ (1). Society for Fetal Urology (SFU) grade 3-4 of postnatal hydronephrosis and relative renal function (RRF) less than $40 \%$ are significant independent predictive factors for surgery. Preservation of renal function is a main goal of follow up of a patient with antenatal hydronephrosis. Although conservative treatment of these patients may spare them unnecessary surgery, it always carries some risk of irreversible loss of renal function. The use of different tissue and urinary markers in the clinical setup allows the diagnosis of urinary obstruction at the early stage therefore avoiding renal parenchymal damage. Previous studies confirmed increased urinary secretion of transforming growth factor- $\beta$ (TGF- $\beta$ ) and epidermal growth factor (EGF) in obstructive uropathy making them attractive markers for early diagnosis of renal parenchymal

\section{EDITORIAL COMMENT}

This study evaluated the utility of U-NAG/ $\mathrm{Cr}$ as a marker for renal obstruction in patients with hydronephrosis. All patients had vesicoureteral reflux ruled out and underwent a well-tempered renogram which was interpreted with fairly strict obstructive criteria (diminished function or $\mathrm{t} 1 / 2>20 \mathrm{~min}$.). The study population included 16 patients with grade 3 hydronephrosis and 12 patients with grade 4 hydronephrosis. There were too few patients with grade $1(n=1)$ and grade 2 hydronephrosis $(n=2)$ to draw any valid conclusions for these groups. Patients $\mathrm{U}-\mathrm{NAG} / \mathrm{Cr}$ ratios were compared to historical reference controls. damage. However, the search for more sensitive markers is needed in order to confirm an obstruction at the earliest level and proceeding with the surgery in order to spare these patients unnecessary diagnostic examinations and avoiding irreversible renal damage. The authors should be congratulated for their efforts to find out a new predictive factor of renal function deterioration. Further studies are needed to elucidate a precise mechanism, which is leading to renal parenchymal damage in patients with UPJ obstruction, which could in turn help develop new diagnostic modalities.

\section{REFERENCE}

1. Chertin B, Pollack A, Koulikov D, Rabinowitz R, Hain D, Hadas-Halpren I, et al.: Conservative treatment of uretero-pelvic junction obstruction in children with antenatal diagnosis of hydronephrosis: Lessons learned after 16 years of follow up. Eur Urol. 2006; 49: 734-9.

Dr. Boris Chertin Department of Urology Shaare Zedek Medical Center Jerusalem, Israel E-mail:bchertin@yahoo.com 
further consideration. It implies that even small degrees of hydronephrosis may adversely affect tubular function beyond our capability to measure. Due to the small numbers of grades 1 and 2, these conclusions are best limited to grades 3 and 4 . Future studies in patients with grades 1-2 hydronephrosis should be performed before concluding that U-NAG/ $\mathrm{Cr}$ is elevated even with low grade hydronephrosis.

A number of questions were left unanswered. They imply that all patients with grade 4 hydronephrosis were obstructed and underwent surgery. In such patients, did U-NAG/Cr levels return to normal after repair? If so, then this would imply that NAG might be a potential marker for resolution of obstruction in patients with persistent hydronephrosis after repair. Was there a difference in U-NAG/Cr levels between those that presented later in life with symptoms and those detected prenatally? Lastly, there is no comment regarding the duration of follow-up in these patients. It is conceivable that a change in U-NAG/Cr over time may correlate with subsequent deterioration.

Unfortunately, we are still searching for the "holy grail" of hydronephrosis management - a highly sensitive, highly specific marker of functionally significant obstruction, which is detectable before radiographic obstruction/ deterioration, or clinical symptoms develop.

Dr. David R. Vandersteen

Pediatric Urologic Surgeon \& Associate Chief of Surgery Children's Hospitals and Clinics of Minnesota Professor of Urology, Mayo Graduate School of Medicine Minneapolis, Minnesota, USA

E-mail:dvandersteen@pediatricsurgicalassociates.com 